\title{
STRONGLY RIGID METRICS AND ZERO DIMENSIONALITY
}

\author{
HAROLD W. MARTIN
}

\begin{abstract}
A metric $d$ is strongly rigid if and only if $d(x, y) \neq d(w, z)$ whenever the doubleton $\{x, y\}$ is not equal to the doubleton $\{w, z\}$. It is shown that a nonempty metrizable space $X$ admits a compatible strongly rigid metric if $X$ has covering dimension zero and has cardinality equal to or less than that of the real line.
\end{abstract}

A metric $d$ is said to be strongly rigid if $d(x, y) \neq d(w, z)$ whenever the doubleton $\{x, y\}$ is not equal to the doubleton $\{w, z\}$. If a metrizable space $X$ admits a compatible strongly rigid metric, then $X$ is said to be eventually strongly rigid. In [2], L. Janos introduced the concept of an eventually strongly rigid space and showed that a nonempty separable metrizable space is zero dimensional if and only if it is eventually strongly rigid. The purpose of this note is to extend part of Janos' theorem by showing that if $X$ is a nonempty metrizable space with covering dimension zero, i.e., $\operatorname{dim}(X)=0$, and if $\operatorname{card}(X) \leqslant c$, the cardinality of the continuum, then $X$ is eventually strongly rigid. It is clear that if $X$ is eventually strongly rigid, then $\operatorname{card}(X) \leqslant$ $c$; also, it is easy to show that if $X$ is eventually strongly rigid, then $\operatorname{ind}(X)=0$, e.g., see [2]. It follows that if $\Re$ is any class of metrizable spaces in which the dimension functions ind and dim coincide (e.g., see [1]), then a space $X$ in $\mathfrak{T}$ is eventually strongly rigid if and only if $\operatorname{dim}(X)=0$ and $\operatorname{card}(X) \leqslant c$. Two questions naturally arise: If $\operatorname{ind}(X)=0$, is $X$ necessarily eventually strongly rigid? If $X$ is eventually strongly rigid, must $\operatorname{dim}(X)=0$ ? We refer to [3] as a general reference on dimension theory.

Although the result established in this paper is a significant extension of Janos' theorem that a separable, zero dimensional metrizable space is eventually strongly rigid, we wish to point out that the fundamental technique of Janos' proof, namely the construction of a compatible (with the usual topology) strongly rigid metric for the Cantor set $C$, plays an important role in the proof below. For the sake of both completeness and clarity, we shall now describe Janos' construction.

Let $C$ denote the Cantor set; $C=\cap\left\{A^{n}\right\}$ where

$$
A^{1}=[0,1]-(1 / 3,2 / 3), \quad A^{2}=A^{1}-[(1 / 9,2 / 9) \cup(7 / 9,8 / 9)] \text {, etc. }
$$

The components of $A^{n}$ are denoted by $C_{1}^{n}, C_{2}^{n}, \ldots, C_{2^{n}}^{n}$. Let $s=\left\{a_{n}\right\}$ be any

Received by the editors February 22, 1977.

AMS (MOS) subject classifications (1970). Primary 54F45, 54E35.

Key words and phrases. Strongly rigid metric, zero dimensional space.

(c) American Mathematical Society 1977 
strictly decreasing sequence of positive reals which converges to 0 and which has the property that

$$
a_{n}>\sum_{k=n+1}^{\infty} a_{k} \text { for } n=1,2, \ldots
$$

Arrange the series $\sum a_{n}$ in the following way:

$$
\begin{aligned}
& a_{1}^{1} \\
& a_{1}^{2}, a_{2}^{2}, a_{3}^{2} \\
& \vdots \\
& a_{1}^{n}, a_{2}^{n}, \ldots, a_{2^{n}-1}^{n}
\end{aligned}
$$

where $a_{1}^{1}=a_{1}, a_{1}^{2}=a_{2}, a_{2}^{2}=a_{3}$, etc. For each $n=1,2, \ldots$ and $x, y \in C$, define $\rho^{n}(x, y)$ in the following way: if $x$ and $y$ are in the same component of $A^{n}$, put $\rho^{n}(x, y)=0$; if $x \in C_{k}^{n}$ and $y \in C_{m}^{n}$, and assuming that $x<y$, then put $\rho^{n}(x, y)=a_{k}^{n}+a_{k+1}^{n}+\cdots+a_{m-1}^{n}$. Finally, define $\rho(s)=\rho$ by $\rho(x, y)$ $=\sum_{n=1}^{\infty} \rho^{n}(x, y)$. We refer the reader to [2] for the proof that $\rho(s)$ is a compatible strongly rigid metric for the Cantor set $C$.

THEOREM. Let $X$ be a metrizable space with $\operatorname{dim}(X)=0$ and $\operatorname{card}(X) \leqslant c$. Let $G$ be any open subset of the positive real numbers such that 0 is a limit point of $G$. Then $X$ has an admissible strongly rigid metric $d$ such that $\operatorname{range}(d) \subset$ $\{0\} \cup G$.

Proof. Let $X$ be any metrizable space such that $\operatorname{dim}(X)=0$ and $\operatorname{card}(X)$ $\leqslant c$, and let $G$ be any open subset of positive real numbers such that $0 \in \operatorname{Bd}(G)$. Evidently $(0, \varepsilon) \cap G \neq \varnothing$ for every $\varepsilon>0$.

Choose a sequence $\left\{J_{n}\right\}$ of open intervals $J_{n}=\left(a_{n}, c_{n}\right)$ satisfying the following conditions:

(i) $b_{n} \rightarrow 0$ where $b_{n}=\left(a_{n}+c_{n}\right) / 2$.

(ii) $c_{n+1}<a_{n}$ for $n=1,2, \ldots$

(iii) $\left[a_{n}, c_{n}\right] \subset G$ for all $n$.

(iv) $\sum_{k=n}^{\infty} b_{k}<c_{n}$ for all $n$.

(v) $a_{n}+\sum_{k=n+1}^{\infty} b_{k}<b_{n}$ for all $n$. The existence of such a sequence $\left\{J_{n}\right\}$ can easily be established inductively, and we therefore omit the details. Let $I_{1}=\left(b_{1}, c_{1}\right]$ and $K_{1}=\left(a_{1}, b_{1}\right)$. Let $I_{n}=\left(b_{n}, c_{n}\right)$ and $K_{n}=\left(a_{n}, b_{n}\right)$ for $n=2$, $3, \ldots$ Let $s=\left\{b_{n}\right\}$ and let $\rho=\rho(s)$ be the Janos metric on the Cantor set $C$ as defined above. Observe that if $x$ and $y$ are distinct points of $C$, then there exists some positive integer $n$ such that $\rho(x, y) \in I_{n}$.

Let $r$ be any admissible metric for the space $X$. If $A \subset X$, let $\operatorname{diam}(A)=$ $\sup \{r(a, b): a, b \in A\}$. Since $\operatorname{dim}(X)=0$ and $\operatorname{card}(X) \leqslant c$, there exists an open basis $\left\{B^{n}(a): n=1,2, \ldots ; a \in C=\right.$ Cantor set $\}$ with the following properties: 
(a) For $n$ fixed, $\left\{B^{n}(a): a \in C\right\}$ is a covering of $X$.

(b) If $a \neq b$ in $C$, then $B^{n}(a) \cap B^{n}(b)=\varnothing$ for all $n$.

(c) If $n>m$ and $a, b \in C$, then either $B^{n}(a) \subset B^{m}(b)$ or $B^{n}(a) \cap B^{m}(b)$ $=\varnothing$.

(d) $\operatorname{diam}\left(B^{n}(a)\right)<1 / n$ for all $a \in C$. We allow the possibility that some of the sets $B^{n}(a)$ are empiy.

Let $C_{n}=C$ for $n=1,2, \ldots$, where each Cantor set is endowed with the relative usual topology. The topological product space $\prod_{n=1}^{\infty}\left\{C_{n}\right\}$ may be topologically embedded in the Cantor set $C$; let $g: \Pi\left\{C_{n}\right\} \rightarrow C$ be a homeomorphism from $\Pi\left\{C_{n}\right\}$ into $C$. Define $h: X \rightarrow \Pi\left\{C_{n}\right\}$ by $h(x)=\left\{x_{n}\right\}$ where $\left\{x_{n}\right\}$ is the unique sequence of elements of $C$ such that $x \in B^{n}\left(x_{n}\right)$ for $n=1,2, \ldots$ The function $h$ is a one-to-one continuous map from $X$ into II $\left\{C_{n}\right\}$. Let $f: X \rightarrow C$ be defined by $f(x)=g(h(x))$ for all $x \in X$. Then $f$ is a one-to-one continuous map from $X$ into the Cantor set $C$. Because $f$ is one-to-one, we may define a metric $\delta$ on the set $X$ in terms of the Janos metric $\rho$ on $C$ by $\delta(x, y)=\rho(f(x), f(y))$. The continuity of $f$ plays an important role at a later stage.

If $x$ and $y$ are distinct points of $X$, we define $\operatorname{order}\{x, y\}=1$ if and only if there exist distinct elements $a$ and $b$ in $C$ such that $x \in B^{1}(a)$ and $y \in B^{1}(b)$. If $x \neq y$ are points of $X$ and $\operatorname{order}\{x, y\} \neq 1$, define order $\{x, y\}=n$ if and only if for each natural number $i<n$ there exists $x_{i}$ in $C$ with $x, y \in B^{i}\left(x_{i}\right)$ and there exist distinct elements $a$ and $b$ in $C$ with $x \in B^{n}(a)$ and $y \in B^{n}(b)$.

We may now define the desired metric $d$ on the set $X$. Let $x$ and $y$ be distinct points of $X$ with $\operatorname{order}\{x, y\}=n$. Let $S_{n}=I_{1} \cup I_{2} \cup \cdots \cup I_{n}$. Then define

$$
d(x, y)= \begin{cases}\delta(x, y) & \text { if } \delta(x, y) \in S_{n} \\ \delta(x, y)+a_{n} & \text { if } \delta(x, y) \notin S_{n}\end{cases}
$$

If $x=y$, let $d(x, y)=0$. From (iii) $-(\mathrm{v})$ above, it is evident that range $(d) \subset$ $\{0\} \cup G$. The proof shall be completed by showing that $d$ is a strongly rigid metric which is equivalent to $r$.

It is clear that $d(x, y)=0$ if and only if $x=y$, that $d(x, y)=d(y, x)$, and that $d(x, y) \geqslant 0$. In order to establish the triangle inequality, it is sufficient to consider three arbitrary distinct points $x, y$ and $z$ of $X$. Suppose first that $\operatorname{order}\{x, y\}=\operatorname{order}\{x, z\}=\operatorname{order}\{y, z\}=n$. We shall show that $d(x, y)+$ $d(y, z) \geqslant d(x, z)$. If $\delta(x, z) \in S_{n}$, then $d(x, z)=\delta(x, z)$ and the inequality holds since $\delta$ is a metric. Therefore suppose that $\delta(x, z) \notin S_{n}$. Then $d(x, z)$ $=a_{n}+\delta(x, z)<b_{n}$ by (ii), (iv) and (v) above. If $\delta(x, y) \in S_{n}$, then $b_{n}<$ $\delta(x, y)$ and the triangle inequality holds; if $\delta(x, y) \notin S_{n}$, then $d(x, y)=$ $\delta(x, y)+a_{n}$, and the inequality holds by virtue of the fact that $\delta$ is a metric. Thus, the triangle inequality holds whenever the orders of the three pairs $\{x, y\},\{y, z\}$ and $\{x, z\}$ are equal.

Now assume that not all the orders of the pairs $\{x, y\},\{y, z\}$ and $\{x, z\}$ are equal. Then there exist positive integers $n<m$ which are the orders of 
two of the pairs, say $\operatorname{order}\{x, y\}=n<m=\operatorname{order}\{x, z\}$. We have $x, y \in$ $B^{i}\left(y_{i}\right)$ for $1 \leqslant i<n$ and $y \in B^{n}\left(y_{n}\right)$ while $x \notin B^{n}\left(y_{n}\right)$; on the other hand, since $\operatorname{order}\{x, z\}=m>n$, we have $x, z \in B^{i}\left(z_{i}\right)$ for $1 \leqslant i<m$; evidently $y_{i}=z_{i}$ for $1 \leqslant i<n$ and $y_{n} \neq z_{n}$, that is, $\operatorname{order}\{y, z\}=n$. In summary, if $x$, $y$ and $z$ are any three distinct points of $X$, and not all of the numbers $\operatorname{order}\{x, y\}, \operatorname{order}\{y, z\}$ and $\operatorname{order}\{x, z\}$ are equal, then there exist two positive integers $n$ and $m$ with $n<m$ such that two of the three orders are equal to $n$ and the third order is equal to $m$.

Suppose that the orders of $\{x, y\},\{y, z\}$ and $\{x, z\}$ are not all equal. There exist integers $n<m$ which are the values of the orders. There are three cases to consider in establishing that $d(x, y)+d(y, z) \geqslant d(x, z)$, namely, the case in which order $\{x, y\}=m$, that in which order $\{y, z\}=m$ and that in which order $\{x, z\}=m$.

Assume that order $\{x, y\}=m$. If $\delta(x, z) \in S_{n}$, then $d(x, z)=\delta(x, z)$ and the inequality holds. Therefore, suppose that $\delta(x, z) \notin S_{n}$. Then $d(x, z)=a_{n}$ $+\delta(x, z)$. If $\delta(y, z) \in S_{n}$, then $b_{n}<\delta(y, z)=d(y, z)$ and $a_{n}+\delta(x, z)<$ $b_{n}$ so that the inequality holds. If $\delta(y, z) \notin S_{n}$, then $d(y, z)=a_{n}+\delta(y, z)$, and the inequality follows from the fact that $\delta$ is a metric. Thus, $d(x, y)+$ $d(y, z) \geqslant d(x, z)$ when $\operatorname{order}\{x, y\}=m$ and $\operatorname{order}\{x, z\}=\operatorname{order}\{y, z\}=$ $n$. The remaining two cases are handled in a similar fashion and we therefore omit the details.

Let $\{x, y\}$ and $\{w, z\}$ be distinct doubleton subsets of $X$. In order to show that $d$ is strongly rigid we must show that $d(x, y) \neq d(w, z)$. In case $d(x, y)$ $=\delta(x, y)$ and $d(w, z)=\delta(w, z)$, we have $d(x, y) \neq d(w, z)$ since $\delta$ is strongly rigid. Suppose $d(x, y)=\delta(x, y)$ and $d(w, z) \neq \delta(w, z)$; then $d(x, y)$ $\in I_{n}$ for some $n$ while $d(w, z) \in K_{m}$ for some $m$; by (ii), $I_{n} \cap K_{m}=\varnothing$ so that necessarily $d(x, y) \neq d(w, z)$. Similarly, if $d(x, y) \neq \delta(x, y)$ and $d(w, z)=$ $\delta(w, z)$, then $d(x, y) \neq d(w, z)$. Finally, suppose that $d(x, y) \neq \delta(x, y)$ and $d(w, z) \neq \delta(w, z)$. If $\operatorname{order}\{x, y\}=n$ and $\operatorname{order}\{w, z\}=m$, then $d(x, y) \in$ $K_{n}$ and $d(w, z) \in K_{m}$; if $n \neq m$, then clearly $d(x, y) \neq d(w, z)$. Suppose $n=m$. Then $d(x, y)=\delta(x, y)+a_{n}$ and $d(w, z)=\delta(w, z)+a_{n}$; since $\delta(x, y) \neq \delta(w, z)$, necessarily $d(x, y) \neq d(w, z)$, and we have established that $d$ is a strongly rigid metric.

We shall complete the proof of the theorem by showing that the metrics $r$ and $d$ are equivalent.

Let $r\left(x_{n}, x\right) \rightarrow 0$. Since $f: X \rightarrow C$ is continuous, we have $\rho\left(f\left(x_{n}\right), f(x)\right) \rightarrow 0$ whence $\delta\left(x_{n}, x\right) \rightarrow 0$. Let $x \in B^{n}\left(y_{n}\right)$ for $n=1,2, \ldots$ Given $\varepsilon>0$, there exists $m$ so that $B^{m}\left(y_{m}\right) \subset\{y: r(x, y)<\varepsilon\}$; by (i) we also may assume that we have chosen $m$ so large that $b_{m}<\varepsilon$. Since $r\left(x_{n}, x\right) \rightarrow 0$ and $\delta\left(x_{n}, x\right) \rightarrow 0$, we may choose a natural number $N$ such that if $n>N$, then $x_{n} \in B^{m}\left(y_{m}\right)$ and $\delta\left(x_{n}, x\right)<\varepsilon$. Let $n>N$ and suppose $\operatorname{order}\left\{x, x_{n}\right\}=j$. If $\delta\left(x, x_{n}\right) \in S_{j}$, then $d\left(x, x_{n}\right)=\delta\left(x, x_{n}\right)$, that is, $d\left(x, x_{n}\right)<\varepsilon$. If $\delta\left(x, x_{n}\right) \notin S_{j}$, then $d\left(x, x_{n}\right)$ $=a_{j}+\delta\left(x, x_{n}\right)$; now we have $x_{n} \in B^{m}\left(y_{m}\right)$ for all $n>N$ so that $m<j$ whence $a_{j}<a_{m}$; but then $a_{j}+\delta\left(x, x_{n}\right)<a_{m}+\delta\left(x, x_{n}\right)<b_{m}<\varepsilon$, that is, 
$d\left(x, x_{n}\right)<\varepsilon$. Thus, $d\left(x, x_{n}\right)<\varepsilon$ whenever $n>N$ so that $d\left(x, x_{n}\right) \rightarrow 0$. Now suppose that $r\left(z_{n}, x\right) \nrightarrow 0$. Choose a subsequence $\left\{x_{n}\right\}$ of $\left\{z_{n}\right\}$ and an $\varepsilon>0$ such that $r\left(x_{n}, x\right)>\varepsilon$ for $n=1,2, \ldots$ Choose $m$ so large that $x_{n} \notin B^{m}\left(y_{m}\right)$ for all $n$. Then $\operatorname{order}\left\{x, x_{n}\right\} \leqslant m$ for all $n$. It follows that $d\left(x, x_{n}\right)>a_{m}$ for all $n$, that is, $d\left(z_{n}, x\right) \nrightarrow 0$. Thus, for any sequence $\left\{x_{n}\right\}$ and point $x$, we have $d\left(x, x_{n}\right) \rightarrow 0$ if and only if $r\left(x, x_{n}\right) \rightarrow 0$, showing that $r$ and $d$ are equivalent metrics, and completing the proof.

\section{REFERENCES}

1. B. Fitzpatrick and R. Ford, On the equivalence of small and large inductive dimension in certain metric spaces, Duke Math. J. 34 (1967), 33-39.

2. L. Janos, A metric characterization of zero-dimensional spaces, Proc. Amer. Math. Soc. 31 (1972), 268-270.

3. J. Nagata, Modern dimension theory, Wiley, New York, 1965.

Department of Mathematics, College of St. Scholastica, Duluth, Minnesota 55811 